\title{
Arthroskopisch assistierte Behandlung von Tibiakopffrakturen
}

\author{
Michael A. Scherer, Sebastian Riha
}

\section{Zusammenfassung}

Tibiakopffrakturen stellen hohe Anforderungen an den Chirurgen. Man unterscheidet Luxationsfrakturen, Plateaufrakturen, Trümmerfrakturen und extraartikuläre Frakturen. Es stehen konservative und operative Therapieoptionen zur Wahl. Welches Verfahren zum Einsatz kommt, ist in erster Linie von der Art der Fraktur und den Weichtverhältnissen sowie von der zu erwartenden Compliance des Patienten abhängig.

In den letzten Jahren etabliert sich die arthroskopisch unterstützte Versorgung, die weniger invasiv als offen chirurgische Techniken sein kann, die genauere Evaluation von Kniebinnenschäden und eine optische Kontrolle des Repositionsergebnisses ermöglicht und nicht zuletzt die Strahlenbelastung für den Patienten und das Personal reduziert. Sie kann helfen, Folgeschäden wie die posttraumatische Arthrose oder Kniegelenkinstabilitäten zu verringern und die Rehabilitationszeit zu verkürzen. Dem stehen der höhere technische, personelle und zeitliche Aufwand gegenüber.

\section{Arthroscopically Assisted Treatment of Fractures of the Tibial Plateau}

Fractures of the tibial plateau represent a major challenge for surgeons. One distinguishes between dislocation fractures, plateau fractures, comminuted fractures and extra-articular fractures. Both conservative and surgical treatment options are available. The choice of treatment depends in the first instance on the nature of the fracture, the condition the soft tissue and the expected patient compliance. In the past few years arthrosocopically supported management has become established as it can be less invasive than open surgery, allows a more exact evaluation of internal injuries to the knee, enables a visual control of the result of repositioning and, last but not least, reduces radiation exposure for patient and personnel. Also, it can help to reduce subsequent damage such as post-traumatic arthrosis or instability of the knee joint and shorten the rehabilitation time. On the other hand, the procedure is more time-consuming and accompanied by higher technical and personnel requirements.

\section{Einleitung}

\section{Wertigkeit der Arthroskopie}

Die Kniegelenksarthroskopie als minimalinvasive Technik hat bei der Versorgung von Tibiakopffrakturen den primären Stellenwert eines Diagnostikums, das über die Möglichkeiten der reinen Bildwandlerdarstellung oder eine Iso-C-3D hinausgeht. Die intraartikulären Weichteile können besser und vollständiger eingesehen und beurteilt wer-

den. Die Arthroskopie wird zum therapeutischen Verfahren, wenn sich während der Operation zusätzliche behandlungsrelevante Informationen über Weichteilverletzungen, wie zum Beispiel Verletzungen an Sehnen, Bändern, Menisken oder Knorpel ergeben. Auch ein Hämarthros und intraartikuläre Knochen- und Knorpelfragmente, welche die Gelenkfunktion beeinträchtigen können, können regelhaft arthroskopisch entfernt werden.

Die Nachteile der arthroskopischen Unterstützung sind neben dem erhöhten Aufwand und den erhöhten Kosten auch die verlängerte Operationsdauer. Der Operateur muss dafür ausgebildet sein, eine Lernkurve ist zu erwarten.

\section{Klinik}

Klinisch ist die Tibiakopffraktur durch eine Schwellung, Erguss, schmerzhafte Bewegungseinschränkung und Hämatomverfärbung gekennzeichnet. Die Symptomatik ist äußerst variabel. Sie kann bei einfachen Frakturen äußerst gering ausgeprägt sein, mit zunehmender Frakturschwere werden Begleitverletzungen häufiger, Unterschenkelkompartmentsyndrome finden sich in ca. 5-10\% aller AO 41B- und C-Frakturen.

Vor Durchführung einer arthroskopisch assistierten Osteosynthese muß ein Kompartmentsyndrom sicher ausgeschlossen sein. Bei fraglichem klinischem Befund ist die Objektivierung durch invasive Kompartmentdruckmessung erforderlich.

\section{Diagnostik}

\section{Klinische Untersuchung}

Neben der Untersuchung des Kniegelenkes müssen auch der Pulsstatus und periphere sensible und motorische Defizite erfasst werden. Sind die peripheren Pulse nicht klinisch tastbar, erfolgt eine Doppler-Untersuchung im Seitenvergleich. Kann so die Durchblutungssituation nicht geklärt werden, muss sich eine Angiografie bzw. DSA anschließen. Arterienverletzungen treten in ca. 0,7\% aller Tibiakopffrakturen auf [1].

Sensible und motorische Defizite können ein Hinweis auf eine strukturelle Nervenverletzung sein. Gefährdet bei Tibiakopffrakturen ist insbesondere der N. peroneus durch eine begleitende hohe Fibulafraktur. Hyp- und Parästhesien sowie Lähmungen können jedoch auch auf ein Kompartmentsyndrom hindeuten. Bei Verdacht muss unbedingt eine Kom- 
partmentdruckmessung erfolgen. Unter 482 eigenen, dokumentierten Tibiakopffrakturen wurde in 7,5\% eine Kompartmentspaltung aufgrund klinischer Symptome oder harter Messdaten erforderlich.

Neben knöchernen Verletzungen des Kniegelenkes sind insbesondere diejenigen Weichteilverletzungen von Bedeutung, welche eine spätere Instabilität des Kniegelenkes zur Folge haben können. Dazu zählen insbesondere die Rupturen der Kreuzbänder und der Kollateralbänder. Bei Patienten jünger als 40 Jahre reißen die Kollateralbänder häufiger intraligamentär, da die Bandansatzregionen äußerst stabil sind, bei älteren Patienten reißen sie eher knöchern aus [1]. Die Stabilitätsprüfung des Kniegelenkes im akuten Stadium ist vor allem wegen der Schmerzhaftigkeit, aber auch wegen Schwellung und Hämatom meist unergiebig. Deshalb wird sie im OP präoperativ erneut durchgeführt. Verletzungen des Kapselbandapparates sind bei minimal dislozierten Frakturen mit ca. 7\% selten, bei Plateaurandfrakturen treten sie in mehr als der Hälfte der Fälle auf [1]. Schädigungen des Innenmeniskus kommen in 2,5\% der Fälle, des Außenmeniskus in $15 \%$ der Fälle vor.

Die Narkoseuntersuchung des Kniegelenkes auf Bandstabilität ist auch bei der Tibiakopffraktur als Routinemaßnahme zu fordern.

\section{Apparative Diagnostik}

Als apparative Basisdiagnostik dient das Röntgenbild des Kniegelenkes in zwei Ebenen, der Zentralstrahl ist auf den Gelenkspalt ausgerichtet. Bei Verdacht auf eine Tibiakopffraktur ist die a.-p.-Aufnahme in $10^{\circ}$ kraniokaudalem Strahlengang anzufertigen (Plateauaufnahme), um das Ausmaß von Impressionsfrakturen sicherer zu beurteilen. Zwei Schrägaufnahmen können die Diagnostik ergänzen.

Ab 41B2-Frakturen aufwärts, sollte eine präoperative CT-Untersuchung regelhaft durchgeführt werden, da im Röntgenbild das Ausmaß der Frakturen oft unterschätzt wird. Die Punktion des Kniegelenkes, nur bei Ruheschmerzen erforderlich, kann weitere Informationen liefern.

Die MRT des Kniegelenkes ist die Methode der Wahl zur nicht invasiven Beurteilung der Verletzungen des Kapselbandapparates und der Menisken. Sie dient auch der Darstellung in der Röntgendiagnostik unsichtbarer Frakturen (bone bruise, Knochenödem), hat aber in der Akutdiagnostik der Tibiakopffraktur keinen besonderen Stellenwert.

Die Tibiakopffraktur wird im konventionellen Röntgenbild in $40 \%$ unterklassifiziert, ab einer AO-Klassifkation B1.2 aufwärts ist eine präoperative $C T$ zur Operationsplanung zu fordern. Die MRT hat keinen Routineplatz in der Akutdiagnostik.
Die früher gebräuchliche Arthrografie des Kniegelenkes ist überflüssig geworden.

\section{Unfallmechanismen}

Tibiakopffrakturen entstehen durch massive Einwirkung direkter und indirekter Gewalt. Durch extremen Valgusstress entstehen beispielsweise Kondylenbrüche, Spaltbrüche und Kantenabrissfrakturen, durch axiale Belastung kommt es zu Impressions- und Kompressionsfrakturen. Kombinationen verschiedener Kräfte führen zu komplexen Spaltkompressionsfrakturen [2].

\section{Klassifikationen der Tibiakopffraktur}

Die Menge an unterschiedlichen Bruchformen an der Tibia verlangt nach Klassifikationen, die einfach genug für die klinische Anwendung sind, das heißt, dass sie die Entscheidung über die richtige Therapie erleichtern. Gleichzeitig müssen sie jedoch differenziert genug sein, um bei Nachuntersuchungen Ergebnisse vergleichen zu können. Die meistverbreitete Klassifikation der Tibiakopffrakturen ist die AO-Klassifikation für Plateaufrakturen und die Moore-Klassifikation für Luxationsfrakturen [4]. Daneben werden noch die Modifikation nach Schatzker und Hohl-Klassifikation verwendet.

\section{AO-Klassifikation [5]}

Die 41-A1-Ausrissfraktur der Eminentia intercondylaris war die erste, routinemä-

Tab. 1 Wahl des Osteosyntheseverfahrens

\begin{tabular}{lll} 
AO-Klassifikation & Implantat 1. Wahl & Implantat 2. Wahl \\
\hline A1 & kanülierte Schraube & 3,5 mm-Schrauben, Nähte \\
\hline A2 & $\begin{array}{l}\text { perkutane, winkel- } \\
\text { stabile Platte }\end{array}$ & UTN, Expert nail, ggf. mit 1/3-Rohr Platte \\
\hline A3 & Hybridfixateur & UTN, Expert nail, ggf. mit monolateralem medialem Fixateur externe \\
\hline B1 & Schrauben 3,5 oder 6,5 mm & T-, L-Platte, Tibiakopfplatte \\
\hline B2 & Schrauben 3,5 oder 6,5 mm & T-, L-Platte, Tibiakopfplatte \\
\hline B3 & $\begin{array}{l}\text { T-, L-Platte, winkel-stab. } \\
\text { Tibiakopfplatte }\end{array}$ & \begin{tabular}{l} 
Zusatzimplantate nach Erfordernissen \\
\hline C1
\end{tabular} \\
\hline T-, L-Platte, winkel-stab. & $\begin{array}{l}\text { PTN Expert nail und Schrauben, Zusatzimplantate nach Erfordernissen } \\
\text { Tibiakopfplatte }\end{array}$ & $\begin{array}{l}\text { perkutane Doppelplatte 3,5 mm, Zusatzimplantate nach Erfordernissen, } \\
\text { Hybridfixateur }\end{array}$ \\
\hline C3 & Tibiakopfplatte & $\begin{array}{l}\text { perkutane Doppelplatte 3,5 mm, Zusatzimplantate nach Erfordernissen, } \\
\text { Hybridfixateur }\end{array}$ \\
\hline
\end{tabular}


ßig arthroskopisch assistiert versorgte Tibiakopffraktur (vgl. Abb. 2-4), die Versorgung von monokondylären, Spaltoder Impressionsfrakturen (B1-B3) und von bikondylären Spalt- oder Impressionsfrakturen blieb Sonderfällen vorbehalten.

\section{Ask-assistierte Osteosynthese}

\section{Operationsziel}

Das Ziel der Osteosynthese des Tibiakopfes ist eine möglichst anatomische Reposition der Gelenkfläche ohne Stufenbildung bei gleichzeitig wiederhergestellter Gelenkachse. Dieses Ergebnis soll dann mit Implantaten übungsstabil gesichert werden. Die Zugänge sollten so gewählt werden, dass eine möglichst effektive Weichteilschonung erreicht wird.

\section{Osteosyntheseverfahren}

Für die verschiedenen Frakturentitäten stehen viele verschiedene Implantate zur Verfügung. Diese sind in Tab. 1 dargestellt

\section{Operationszeitpunkt}

Grundsätzlich gilt es, eine frühestmögliche Frakturversorgung anzustreben. Einfluss auf den Zeitpunkt der Versorgung hat die Weichteilsituation, das Ausmaß des geschlossenen oder offenen Traumas, der Frakturtyp und Begleitverletzungen, insbesondere Verletzungen der Blutgefäße und Nerven.

Lagerung und Abdeckung zur arthroskopisch assistierten Osteosynthese

Für eine arthroskopisch assistierte Frakturversorgung geeignete Frakturen, also typischerweise A1.3, B1, B2, B3, C1 und in gewissem Umfang noch C2-Frakturen,

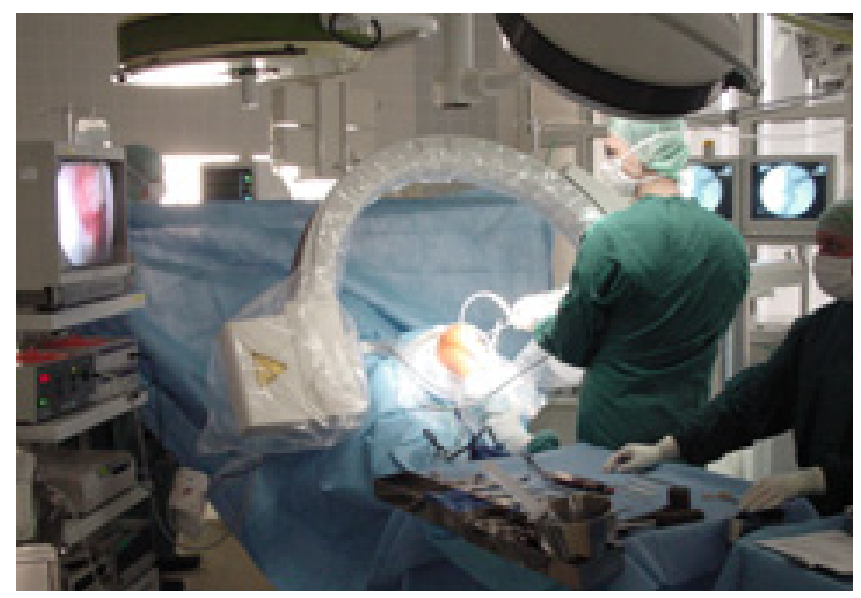

werden in Rückenlage operiert: Das verletzte Bein ist mit einem Tourniquet weit proximal am Oberschenkel armiert und in einer Beinhalterung in knapp $90^{\circ}$ Flexion gelagert. Falls zur Unterfütterung der Fraktur eventuell eine Spongiosaentnahme notwendig sein könnte, sollte der kontralaterale Beckenkamm ebenfalls mit abgedeckt werden.

Ein typischer Aufbau ist in Abb. 1 dargestellt.

\section{Operationstechnik}

Die arthroskopische Versorgung der Eminentia-Ausrißfraktur lässt sich technisch am einfachsten in Analogie zur Kreuzbandplastik mit den zu diesem Zweck verwendeten Zielgeräten bewerkstelligen.

Nach Einleitung der Narkose erfolgt zunächst die erneute Untersuchung des verletzen Kniegelenkes auf Bandinstabilitäten. Stressaufnahmen unter Bildwandlerkontrolle dokumentieren solche Defizite und Läsionen.

Je gravierender die Verletzung ist, desto wichtiger ist es, zur Vermeidung eines iatrogenen Kompartmentsyndroms zuerst den Zugang zum Gelenk zu präparieren und erst anschließend zu arthroskopieren. Hierbei muss von Anfang an ein freier Abfluss aus dem Gelenk gewährleistet sein. Besonderes Augenmerk auf immer möglichen Paravasate ist bei lateral nicht begrenzten und in die tiefe Beugerloge ausstrahlende Frakturen zu lenken.

Bei allen arthroskopisch unterstützten Osteosynthesen ist unbedingt auf freien Abfluß der Spülflüssigkeit und die Vermeidung von Paravasate zu achten.

Abb. 1 Eine Bildwandlereinstellung in „HWS-Position“ unter Verzicht auf eine intraoperative Durchleuchtung im a.-p.-Strahlengang ermöglicht eine simultane radiologische und arthroskopische Kontrolle des operativen Vorgehens.
Die Standard-Indikation ist die Eminentia-Ausrissfraktur. Auch radiologisch beeindruckende Befunde können intraartikulär durch den Hoffaschen Fettkörper und gedehnte Kreuzbandanteile versteckt liegen. Der Fettkörper sollte mit dem Shaver oder einem Elektrokauter (arthrocare oder ähnlichem) soweit reseziert werden, dass eine eindeutige Übersicht möglich ist.

Im eigenen Vorgehen (Abb. 2,3) hat sich folgendes Prozedere bewährt: Shaving zur Darstellung der Fraktur, Identifikation von sichtbaren Frakturlinien und Verwerfungen, Stichinzision kraniomedial in Patellamitte und Vorschieben eines $2 \mathrm{~mm}$ Drahtes an der medialen Femuropatellargelenks-Facette vorbei auf die Eminentia, Manipulation des knöchernen Fragmentes und Fixation mit einem VKB-Zielgerät über den normalen anteromedialen Zugang. Jetzt lassen sich sequenziell Gewinde-Kirschner-Drähte für kanüllierte Schrauben einbringen. Die Gewinde-Kirschner-Drähte sind arthroskopisch in der Regel auch nach Perforation der Gelenkbinnen-Kortikalis der Eminentia nicht zu sehen, sondern durch das VKB maskiert, eine simultane Röntgenkontrolle ist zweckmäßig. Die von anteromedial distal eingeführten Schrauben (Bohrkanalrichtung für die VKBPlastik) lassen sich durch eine von anteromedial proximal zusätzlich eingebrachte Schraube stabilisieren (Abb. 3). Alternativ können selbstverständlich auch arthroskopische Schlingen- und Nahttechniken, Auszug der Fäden über tibiale Bohrlöcher und Knoten über einer tibialen Knochenbrücke oder über Fadenankern/Beilagscheiben als Technik angewendet werden.

Selbst bei komplexen Verletzungsformen (Abb.4) weist diese Technik eine ausreichende Stabilität für eine frühfunktionelle Nachbehandlung auf. In klinischen Nachuntersuchungen mit instrumenierter Laxizitätsmessung ist eine radiologisch bestimmte, knöcherne Konsolidierung der Eminentiafraktur keine Conditia sine qua non für eine funktionell hochwertige Gelenksstabilität. Isolierte Ausrissfrakturen des Ansatzes des hinteren Kreuzbandes stellen eine Indikation für einen Eingriff in Bauchlage und eine anatomische Refixation über einen kurzstreckigen hinteren Zugang dar.

In der täglichen Praxis überwiegen in den meisten Statistiken die B-Frakturen, die einer arthroskopisch assistierten Versorgung auch am leichtesten und 


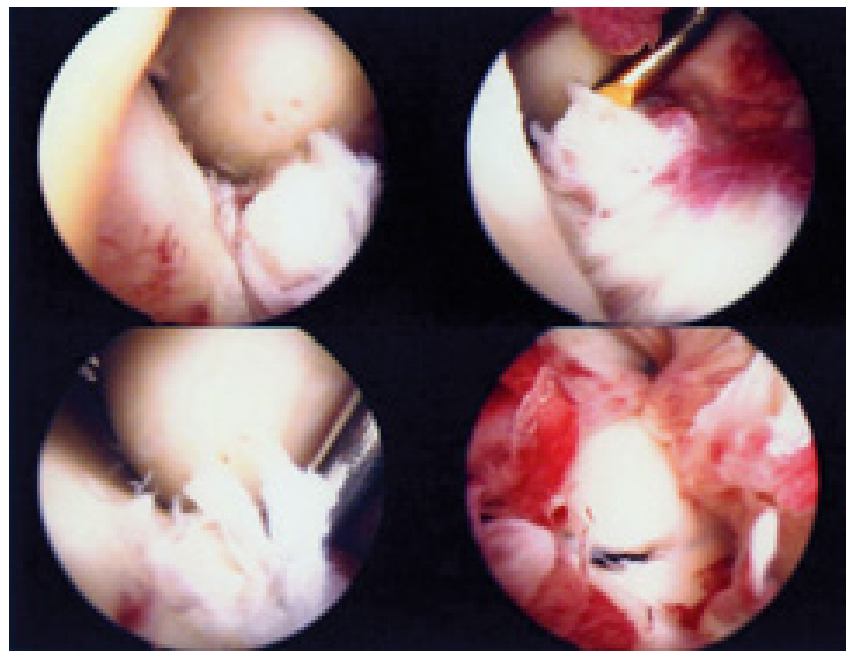

Abb. 2 Präparationsgang bei der Eminentia-Fraktur: links oben - Shaving zur Darstellung der Fraktur; rechts unten - Identifikation von sichtbaren Frakturlinien und Verwerfungen, Stichinzision kraniomedial in Patellamitte und - rechts oben - Vorschieben eines $2 \mathrm{~mm}$ Drahtes an der medialen Femuropatellargelenks-Facette vorbei auf die Eminentia, Manipulation des knöchernen Fragmentes und - links unten - Fixation mit einem VKB-Zielgerät über den normalen anteromedialen Zugang. Jetzt lassen sich sequenziell Gewinde-Kirschner-Drähte für kanüllierte Schrauben einbringen.
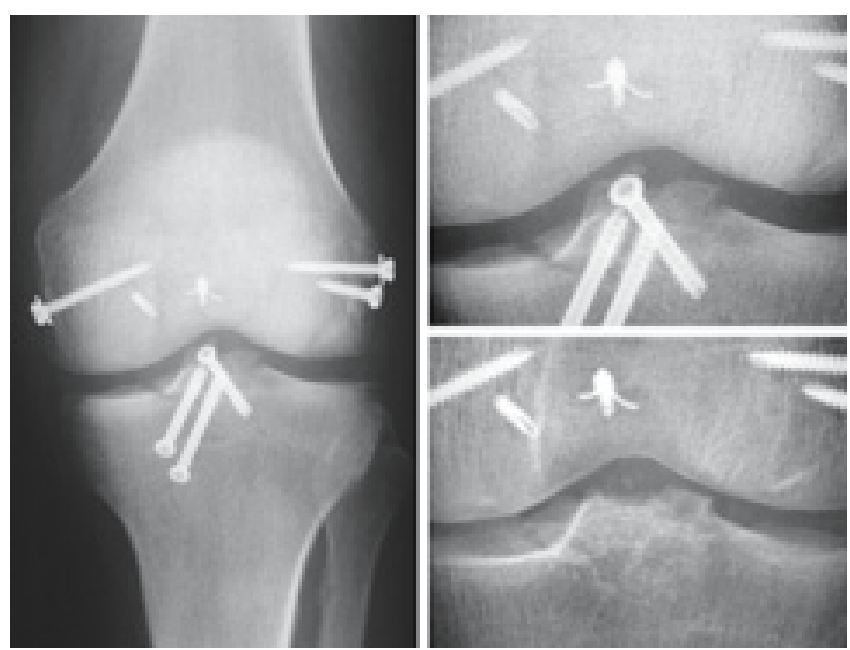

Abb. 4 Kniegelenksluxation mit AO41-A1.3 Eminentia-Ausrissfraktur, femoralem Ausriss des hinteren Kreuzbandes und Abriss beider Kollateralbänder im Rahmen eines Motorrad-Unfalls. Einseitige Rekonstruktion aller rupturierten Bänder mit nicht resorbierbarem Nahtmaterial, typische Anordnung der Eminentia-Verschraubung.

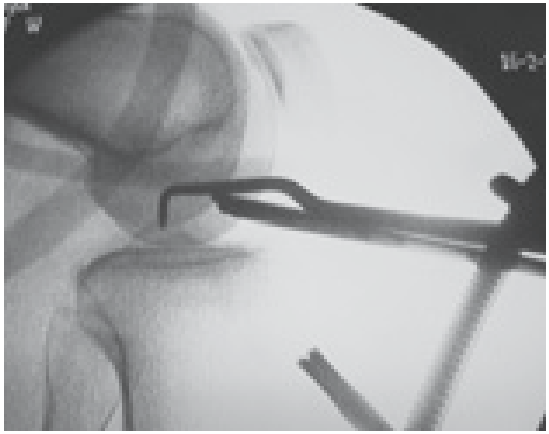

Abb. 3 Intraoperative Durchleuchtung bei reponiertem Eminentia-Fragment. Bei knöcherner Fragmentation Auszugsnähte.

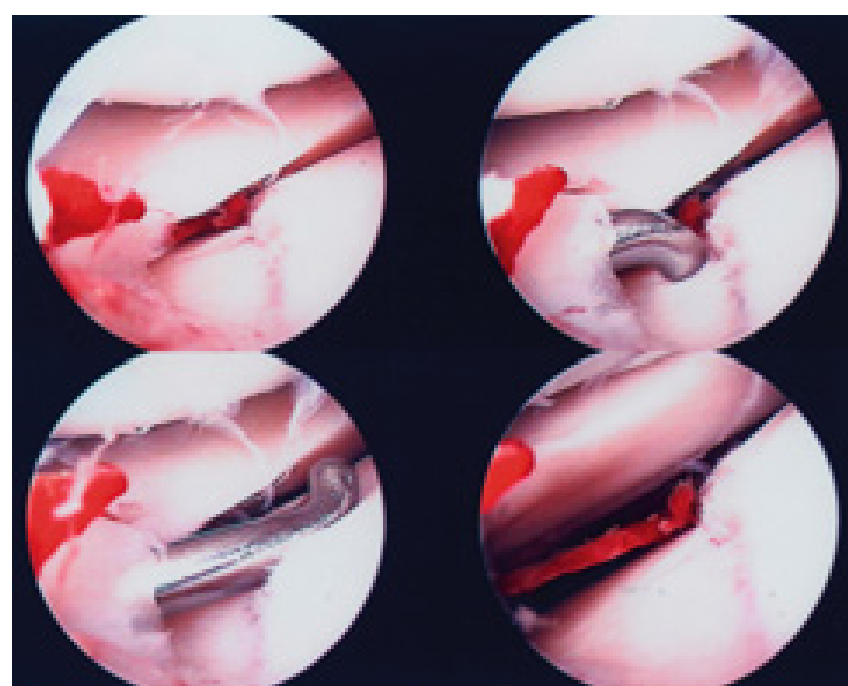

Abb.5 Arthroskopisches Bild nach Lavage des Hämarthros mit gut sichtbarer Stufe nahe dem freien Rand des Außenmeniskus (links oben); bei Druck auf den Knorpel am lateralen Fragment deutlicher, fester Widerstand (rechts oben), die Stufe egalisiert sich aber bei Druck mit dem Tasthaken auf den zentralen Frakturanteil. Keine Indikation zur weitergehenden Reposition . sichersten zugänglich sind. Reine Impressionsfrakturen lassen sich mit einem Tasthaken einfach und reproduzierbar hinsichtlich der Stufenbildung, Stabilität von Knorpelfragmenten und Verwerfungen kontrollieren (Abb.5).

Reine Spaltbrüche werden zunächst unter arthroskopischer Kontrolle reponiert, sehr gut geeignet ist eine große Beckenzange mit Oliven, um ein Einbrechen der Zange in die weiche Tibiaepiphyse zu vermeiden, und anschließend temporär mit Kirschner-Drähten fixiert, die dann durch Großfragmentschrauben ersetzt werden. Die Schrauben sollen knapp unterhalb der Gelenkfläche zu liegen kommen (Abb. 6, 7).
Die arthroskopische Technik darf nicht dazu verleiten, eine mechanisch insuffiziente Osteosynthese aus dann falsch verstandener „minimaler Invasivität“ anzuwenden. Art, Technik und Umfang der Osteosynthese hängen vom Charakter und den biomechanischen Eigenschaften der Fraktur ab und nicht von der Verwendung der Arthroskopie. 
Die „arthroskopisch assistierte Osteosynthese" darf nicht zu einer Änderung der biomechanischen Prinzipien der Osteosynthesetechnik - also beispielsweise Schrauben statt Abstützplatte führen.

Die Technik der Interferenzschraubenosteosynthese kommt aus der Revisionschirurgie des vorderen Kreuzbandes und lässt sich für lokalisierte B2-Frakturen einsetzen: Unter arthroskopischer Kontrolle wird durch einen Kreuzbandzielbügel ein Bohrdraht in der Mitte des Fragmentes platziert. Anschließend erfolgt durch eine Inzision eine Bohrung mit einem Hohlbohrer bis unmittelbar unterhalb des Tibiaplateaus, möglichst ohne Knochenverlust. Mit einem Stößel, der so geformt ist, dass er durch den Bohrkanal parallel zum Tibiaplateau liegt, wird das Fragment hochgestößelt.
Zuletzt wird eine resorbierbare Interferenzschraube eingebracht. Ihre Länge wird vom komprimierten Knochen bis zur tibialen Austrittsstelle bemessen, ihre Dicke entspricht der Dicke des Hohlbohrers plus zwei Millimeter, bei osteoporotischem Knochen plus drei Millimeter.

Lateral nicht begrenzte Frakturen, die eine Tendenz zur Fragmentation aufwei-
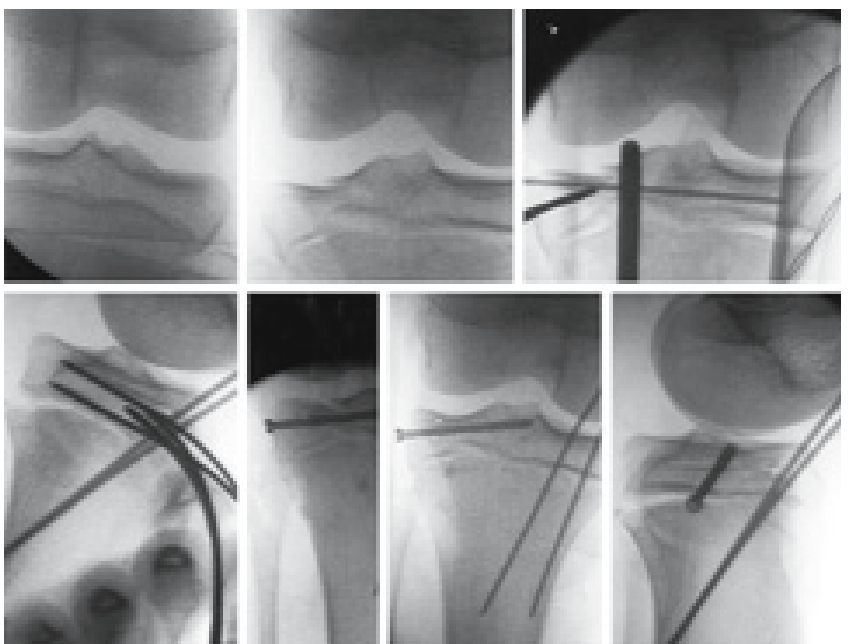

Abb. 6 Beispiel für eine minimalinvasive Osteosynthese beim Kind, technisch schwierige gedeckte Reposition mit Joysticks. Nach arthroskopisch assistierter Fixation epiphysäre Verschraubung und transepiphysäre Kirschner-Drahtung.
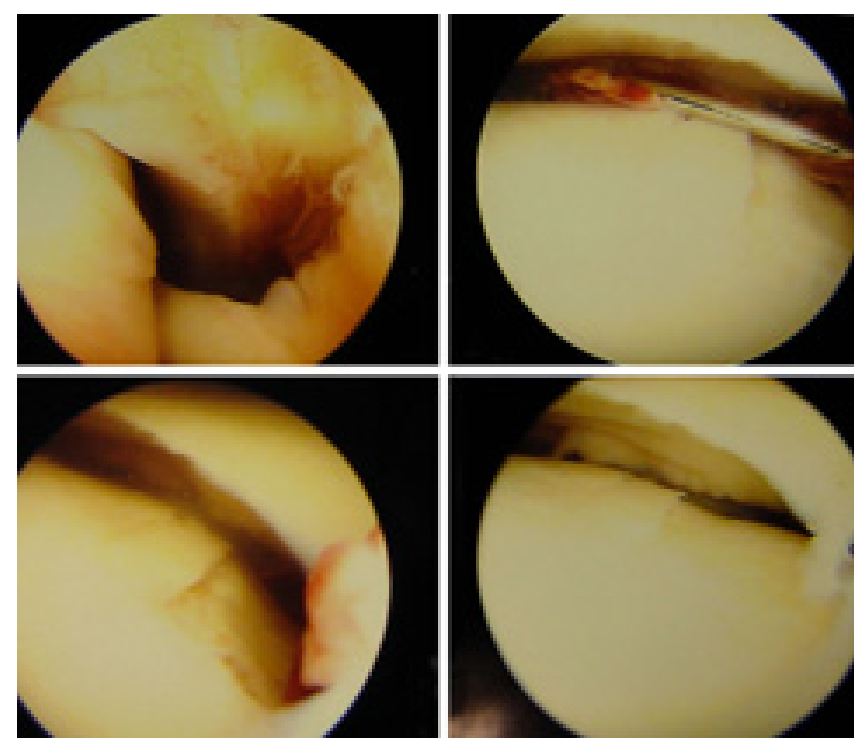

Abb. 8 Lateral nicht begrenzte Impressionsfraktur. Links oben pathologisch weiter Popliteusschlitz, links unten Kantenabbruch unter dem Außenmeniskus. Rechts oben intraartikulärer Kirschner-Draht als Lineal zur Niveaubestimmung, beginnende und rechts unten abgeschlossene Reposition des Kantenfragmentes.

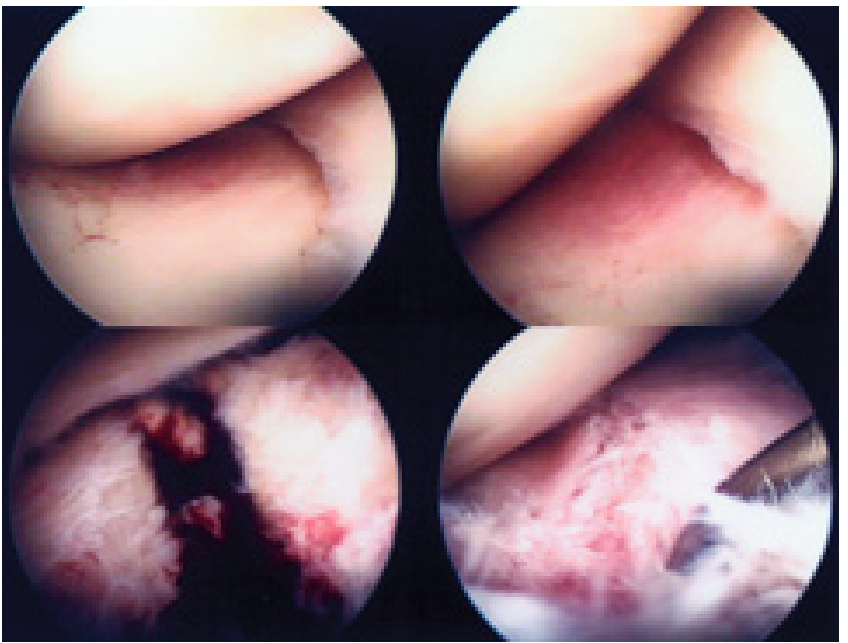

Abb. 7 Erhebliches Weichteiltrauma, partielle Outerbridge IV-Läsion mit vollständiger Knorpelglatze, Knorpelkontusion und Einblutung im medialen Kompartment (obere Reihe) und laterale Spaltfraktur links vor und rechts nach arthroskopisch kontrollierter Reposition (untere Reihe).

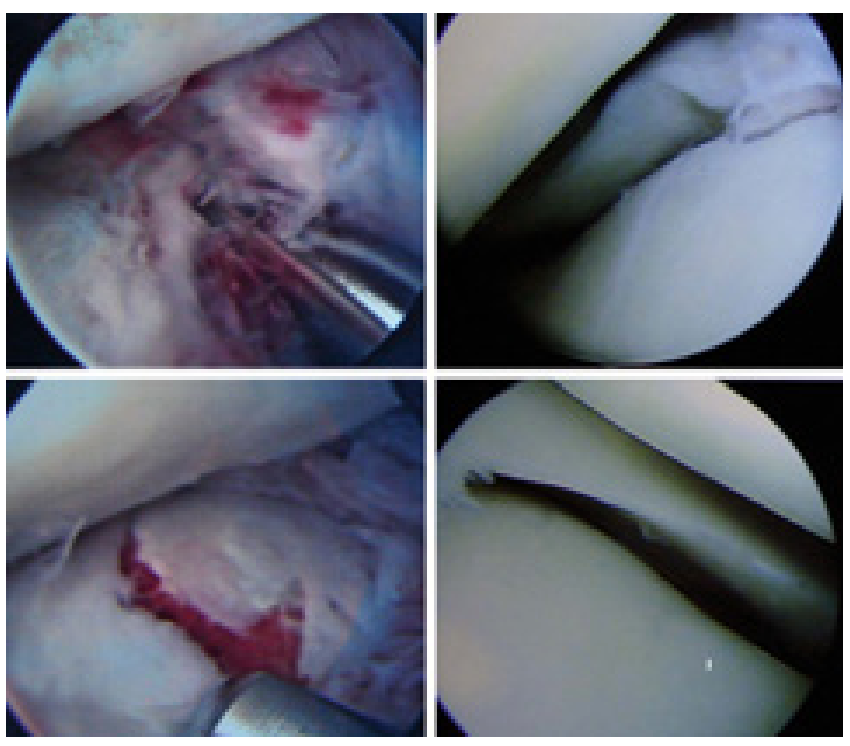

Abb. 9 Pat. BF, zum Unfallzeitpunkt 31 Jahre alt; links intraoperativer Situs am 03.03.2005, Spalt-Impressionsbruch, oben: Aufspleißung des Außenmeniskus-Hinterhorns, Knorpelschaden Outerbridge III; unten: sternförmige Impressionsfraktur, Shaverkopf. Rechts intraoperativer Situs bei der Kontroll-Arthroskopie im Rahmen der Metallentfernung 25 Monate später, am 24.04.2007, oben: Außenmeniskushinterhorn, Lig. meniscotibiale laterale posterius mit minimalen narbigen Umwandlungen; unten: diskrete, längsverlaufende Knorpelzeichnung, ansonsten keine Frakturfolgen erkennbar. 
sen, lassen sich mit einem Osteotom „en bloc" anheben, brauchen dann aber einen bikortikelen oder trikortikalen Beckenkammspan zu Abstützung. Das entsprechende arthroskopische Bild findet sich in Abb. 8.

Komplexere Frakturformen müssen nicht notwendigerweise in eine Früharthrose münden (Abb.9), die Aufklärung des Patienten sollte jedoch bei allen intraarti- kulären Frakturen, die über reine Spaltverletzungen hinausgehen, den Hinweis auf eine beschleunigte degenerative Veränderung des Gelenkes beinhalten. Das gilt auch bei optimaler knöcherner Reposition.

Mehrfragmentäre Spalt-Impressionsfrakturen schließlich können sowohl retrograd intrafokal, also aus dem Frakturspalt heraus (Abb. 10), aus isolierten kor- tikalen Knochenfenstern, als auch von intraartikulär her manipuliert werden (Abb. 11).

\section{Fallstricke und Komplikationen}

Die Metaanalyse von Literatur zur Tibiakopffraktur zeigt eine Gesamtkomplikationsrate von bis zu 32\%. Darunter finden sich an erster Stelle Hämatome, dann folgen Hautnekrosen, Phlebothrombosen,
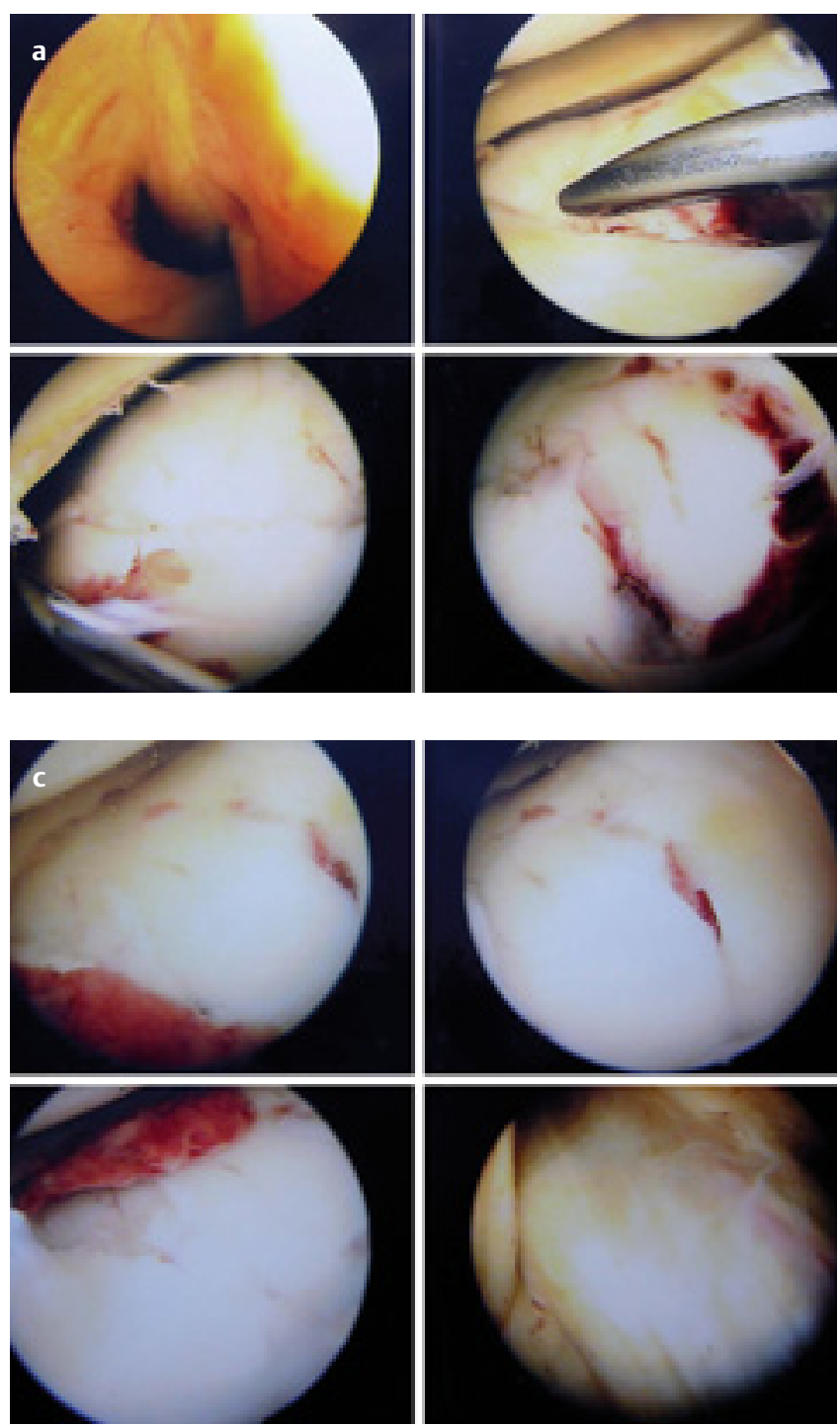
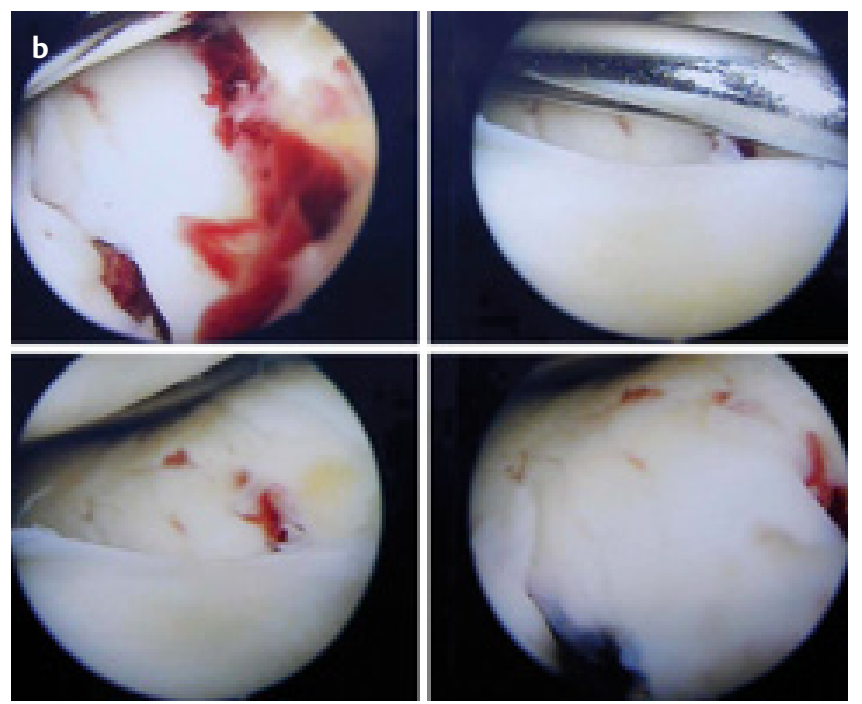

Abb.10 (a) Pat. IL, 28 Jahre alt, Spalt-Impressionsbruch. Links oben: Das intraartikuläre Screening muss das Popliteuseck mit einschließen. Die Mehrzahl der Tibiakopffrakturen liegt lateral, die Mehrzahl aller Weichteilverletzungen bezieht sich auf den Außenmeniskus und seine Bandstrukturen. Bei Verletzungen der meniskopoplitealen Bänder ist mit einer diskreten posterolateralen Rotationsinstabilität zu rechnen, die eventuelle Gelenksinkongruenzen pathophysiologisch betonen können. Rechts oben: ein Tasthaken oder ein Klemmchen helfen bei der Einschätzung von Größen- und Abstandsverhältnissen, bei der Beurteilung von Gelenksstufen oder Spalten. Links unten: Unterfahren des Meniskus, Testung der Stabilität an der Basis und Inspektion des Knorpels unter dem Meniskus. Rechts unten: Darstellung der Fraktur mit Eisschollen-artigem Imprimat. Vor der Reposition sollte mit dem Shaver oder anderen geeigneten Instrumenten die Fraktur gesäubert und einzelne Fragmente taktil auf ihre Beweglichkeit und Stabilität getestet werden. (b) Links oben: zentrales, neben der Eminentia gelegenes Imprimat, bei 1000 bis 12002 mm Kirschner-Draht intraartikulär als interne Höhenkontrolle. Rechts oben: mit Wischbewegungen gelingt es mit dem Draht als Joystick, die Lagebeziehung zwischen Meniskus, Fraktur und Normalhöhe des Gelenksspaltniveaus darzustellen. Untere Reihe: Das eigentliche Ausmaß der Fraktur wird oft durch den Meniskus verdeckt (links). Erst nach Weghalten des Meniskus oder Refixation einer abgerissenen Meniskusbasis stellt sich das Frakturausmaß richtig dar. (c) Status nach Reposition, vgl. auch mit den beiden vorhergehenden Abbildungen. Die Rissbildung im Knorpel ist nur noch andeutungsweise zu erkennen. Abschließend werden Koagel und flottierende Synovialefzen reseziert. Rechts unten: hypovaskuläres VKB, klinisch und auf Tasthakenzug stabil. 
Reizergüsse, oberflächliche Infektionen und Pneumonien.

Die häufigsten technischen Fehler bei der arthroskopisch assistierten Osteosynthese sind

- Arthroskopie vor ausreichendem Zugang, also Ablauf und Paravasate im Weichteil

- Ungenügende intraartikuläre Übersicht

- Verzicht auf mechanisch stabile Osteosyntheseverfahren zugunsten einer (vermeintlichen) geringeren Invasivität

- Versuch, bei radiologisch (Bildwandler) schon „gutem“ Ergebnis das makroskopisch noch befremdliche arthroskopische Bild zu „verbessern“

Erst die Weichteile, dann den Knochen rekonstruieren: Ein abgerissner Meniskus muss erst arthroskopisch genäht oder refixiert werden, sonst ist keine adäquate Reposition möglich.

Bei freiem Abfluss der Spülflüssigkeit kann mit einem maximalen Flow und intraartikulären Drücken bis $80 \mathrm{mmHg}$ gefahrlos gearbeitet werden.

\section{Nachbehandlung}

Übungsstabil versorgte Tibiakopffrakturen werden ab dem 1. p.op. Tag auf eine Bewegungsschiene gelagert und das Bewegungsausmaß auf das maximal für den Patienten tolerable Maß eingestellt. Der Bewegungsumfang über eine ROM von $\mathrm{E} / \mathrm{F}$ 0-0-70/90 richtet sich nach den rekonstruierten Weichteilen (Meniskusnähte).

Im eigenen Vorgehen wird niemals das Kniegelenk selber, sondern ausschließlich das am stärksten in Mitleidenschaft gezogene Kompartment mit einer Überlauf-/Schwerkraft-Drainage drainiert.

Nach Drainagenentfernung ist Sohlenkontakt bis $20 \mathrm{~kg}$ erlaubt - „eine Ameise soll die Belastung überleben“. Fahrradergometer mit minimalem Widerstand und hoher Frequenz ist ebenso wie Gehen im Bewegungsbad im halstiefen Wasser ab der 4. p.op. Woche erlaubt. Eine regelrechte Röntgenkontrolle nach 6 Wochen bietet die Voraussetzung für eine rasche Belastungszunahme nach klinischer Symptomatik. Manuelle Lymph-
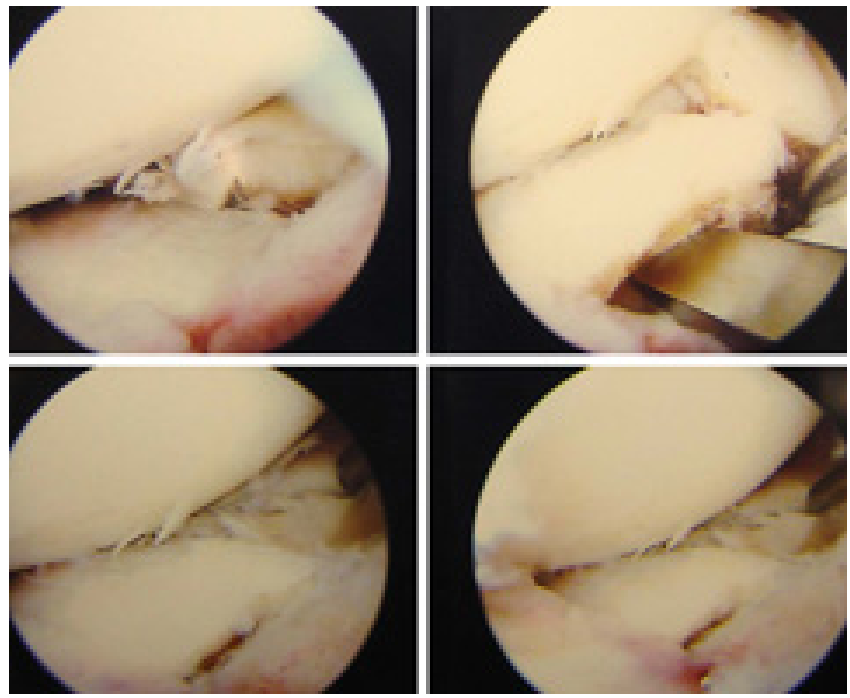

Abb. 11 Komplexes Frakturmuster, Mosaikstein-artiges Einpassen von wichtigen, Gelenktragenden Fragmenten. Hier stößt die arthroskopisch assistierte Osteosynthese dann ebenso an eine Grenze wie ein offenes Verfahren, die eigene Unvollkommenheit liegt allerdings deutlicher zutage.

drainage kann hilfreich sein, sollte aber mit Kompressionsstrümpfen der Klasse II kombiniert werden.

\section{Medikamentös}

Analgetika sollten großzügig verordnet werden, um eine effektive Beübung zu ermöglichen. Eine Antikoagulation mit niedermolekularem Heparin unter Kontrolle der Thrombozyten ist bis zum Erreichen der Vollbelastung durchzuführen, um das Risiko für Thrombosen und Lungenembolien $\mathrm{zu}$ vermindern. Liegt eine Osteoporose vor, so muss eine Osteoporosetherapie nach den Leitlinien veranlasst werden.

\section{Schlussfolgerung}

Der routinemäßige Einsatz der Arthroskopie bei der Tibiakopfosteosynthese ist mit erhöhtem hohen personellen und materiellem Aufwand verbunden, die Operationszeit allein wegen der aufwendigeren Vorbereitung meist etwas verlängert.

Der Einsatz der Arthroskopie bei der Osteosynthese von Tibiakopfosteosynthesen stellt aber eine sinnvolle Ergänzung des Behandlungsspektrums dar: Die Möglichkeit der optischen Kontrolle verleiht dem Operateur eine größere Sicherheit als die isolierte Bildwandlerkontrolle. Vielleicht können durch eine höhere Repositionsgenauigkeit und die initiale
Behandlung von ansonsten okkulten Begleitverletzungen die Spätfolgen der Fraktur abgeschwächt werden.

\section{Literatur}

1 Hohl M. Tibial Plateau Fractures. W.B. Saunders Company, 1997

2 Kennedy JC, Bailey WH. Experimental tibialplateau fractures. SO-A J.Bone Joint Surg. Am. 1968; 1522-1534 Ref Type: Magazine Article

3 Lubowitz J. H, Vance K. J, Ayala, M., Guttmann, D., and Reid, J. B., III: Interference screw technique for arthroscopic reduction and internal fixation of compression fractures of the tibial plateau. Arthroscopy 2006; 22: 1359-1363

4 Moore TM. Fracture-Dislocation of the knee. Clin.Orthop. 1981; 156: 128-140 Ref Type: Magazine Article

5 Müller ME, Allgöwer M, Scheider R, Willenegger H. Manual der Osteosynthese. Springer Verlag Berlin Heidelberg 1992; 3. Auflage: 142-143

6 Scherer MA Arthroskopisch unterstützte Behandlung von Tibiakopffrakturen. Möglichkeiten und Grenzen. Trauma Berufskrankh 2002; 4: 51-62

\section{Prof. Dr. med. Michael A. Scherer Chefarzt}

Chirurg, Unfallchirurg und Orthopäde Dr. med. Sebastian Riha

Assistenzarzt

Abteilung für Unfallchirurgie und Orthopädie Amperkliniken Krankenhausstr. 15 85221 Dachau 